\title{
CAPTURE OF COOLING ELECTRONS BY COOL PROTONS
}

\author{
M. BELL and J. S. BELL \\ CERN 1211 Geneva 23, Switzerland
}

(Received April 20, 1981)

\begin{abstract}
Simplified formulae for the capture of low-energy electrons by stationary protons are averaged over Maxwellian and "flattened" Maxwellian electron velocity distributions. The latter distribution is more nearly appropriate for electron beams used in accelerator proton-beam cooling experiments. Flattening increases the capture rate by a factor of about two. Similar formulae for the capture of antiprotons by protons are mentioned.
\end{abstract}

\section{INTRODUCTION}

In electron cooling of proton beams, ${ }^{1}$ a proton sometimes captures an electron and proceeds as a neutral hydrogen atom. The observation of such atoms yields information about electron and proton beams. ${ }^{2}$ The process is most important in the late stages of cooling when the protons are nearly at rest in the electron gas. The theory of electron capture by protons has of course been worked out. ${ }^{3}$ It results in elaborate expressions requiring computer evaluation. Here we use simplified approximate formulae, adequate for the low relative electron-proton energies of particular interest in electron cooling of accelerator proton beams, that are easily integrated over Maxwellian and "flattened Maxwellian" electron velocity distributions.

\section{FIRST APPROXIMATION}

Spitzer ${ }^{4}$ quotes for the capture cross section of a stationary nucleus for electrons of nonrelativistic velocity $V_{e}$

$$
\sigma\left(V_{e}\right)=A \sum_{n=1}^{\infty} \frac{v_{0}}{v_{n}} \frac{h v_{0}}{E} \frac{g_{n}}{n^{3}},
$$

where $A=2^{4} 3^{-3 / 2} h e^{2} /\left(m_{e}{ }^{2} c^{3}\right)=2.11 \times 10^{-22}$ $\mathrm{cm}^{2} ; h$ is Planck's constant; $e$ is the electron charge; $m_{e}$ is the electron mass; $c$ is the velocity of light; $h v_{0}=Z^{2} \alpha^{2} m_{e} c^{2} / 2$ is the ground state binding energy, $13.6 \mathrm{eV}$ for hydrogen; $h v_{n}=$ radiated photon energy in capture to level $n=h v_{0} /$ $n^{2}+E$; the electron kinetic energy $E=m_{e} V_{e}^{2} /$ $2 ; \alpha$ is the fine-structure constant $=1 / 137$; and $g_{n}$ "is a correction factor, generally about equal to one." For the moment, we follow Spitzer in setting

$$
g_{n}=1
$$

Then

$$
\sigma=A\left(\frac{h v_{0}}{E}\right)^{2} \sum_{1}^{\infty} \frac{1}{n\left(n^{2}+h v_{0} / E\right)} .
$$

That is [see, for example, Abramowitz and Ste$\left.\operatorname{gun}^{5}(6.3 .16)\right]$,

$$
\begin{aligned}
\sigma= & \frac{A}{2} \frac{h \nu_{0}}{E}\left\{\psi\left(1+i \sqrt{\frac{h \nu_{0}}{E}}\right)\right. \\
& \left.+\psi\left(1-i \sqrt{\frac{h \nu_{0}}{E}}\right)+2 \gamma_{1}\right\},
\end{aligned}
$$

where $\psi$ is the digamma function

$$
\psi(z)=(\Gamma(z))^{-1}(d / d z) \Gamma(z),
$$

and $\gamma_{1}$ is Euler's constant

$$
\gamma_{1}=\gamma \cong 0.5772 \text {. }
$$

Later we will give $\gamma_{1}$ a different value. Using the asymptotic form of $\psi$ [Abramowitz and Stegun ${ }^{5}$ (6.3.18)], then

$$
\left(E /\left(h \nu_{0}\right)\right)_{\sigma}=A\left[\gamma_{1}+\ln \vee\left(h \nu_{0} / E\right)\right],
$$

neglecting terms of order $E /\left(h v_{0}\right)$ on the right. The expression is accurate in so far as

$$
E /\left(h v_{0}\right) \ll 1 .
$$


For $E /\left(h v_{0}\right)$ about 0.2 the error is about one per cent.

The rate of capture per proton is

$$
\alpha_{r} n_{e},
$$

where $n_{e}$ is the number of electrons per unit volume and $\alpha_{r}=\left\langle V_{e} \sigma\left(V_{e}\right)\right\rangle$, where the angular brackets denote averaging over the electron velocity distribution.

If one says simply that the distribution is characterized by some typical thermal velocity $V_{T}$ and takes $V_{e}=V_{T}$ everywhere, then from (6)

$$
\alpha_{r}=A Z^{2} \alpha^{2} c^{2} V_{T}^{-1}\left[\dot{\gamma}_{1}+\ln \left(Z \alpha c / V_{T}\right)\right] .
$$

For very small $V_{T}$, the constant $\gamma_{1}$ is negligible in comparison with the logarithm. Then

$$
\alpha_{r} \cong 19.4 Z^{2} \alpha r_{e}^{2} c^{2} V_{T}^{-1} \ln \left(Z \alpha c / V_{T}\right)
$$

A formula equivalent to this is quoted by Budker and Skrinsky ${ }^{6}$ [their Eq. (2.9)]. We try now, at least for well-defined thermal distributions, to be a little more accurate.

\section{SECOND APPROXIMATION}

We consider the Maxwell distribution

$$
f\left(\mathbf{v}_{e}\right)=\left(2 \pi k T / m_{e}\right)^{-3 / 2} \exp (-E /(k T))
$$

and the corresponding "flattened" distribution

$f\left(\mathbf{v}_{e}\right)=\left(2 \pi k T / m_{e}\right)^{-1} \exp (-E /(k T)) \delta\left(V_{e x}\right)$,

obtained by suppressing one of the components of velocity. Then

$$
\begin{aligned}
\alpha_{r} & =\left\langle V_{e} \sigma\left(V_{e}\right)\right\rangle \\
& =\frac{\int_{0}^{\infty} d V_{e} V_{e}^{n-1} V_{e} \sigma\left(V_{e}\right) \exp (-E /(k T))}{\int_{0}^{\infty} d V_{e} V_{e}{ }^{n-1} \exp (-E /(k T))},
\end{aligned}
$$

where $n=3$ for Maxwell and $n=2$ for "flattened." With

$$
z=E /(k T)
$$

as integration variable and using (6),

$$
\begin{aligned}
& \alpha_{r} \vee\left(m_{e} k T / 2\right) /\left(h v_{0} A\right) \\
& =\int_{0}^{\infty} d z z^{n / 2-1} z^{-1 / 2} e^{-z} \\
& \frac{\times\left(\gamma_{1}+1 / 2 \ln \left(h v_{0} /(k T)\right)-1 / 2 \ln z\right)}{\int_{0}^{\infty} d z z^{n / 2-1} e^{-z}} \\
& =\left\{\gamma_{1}+1 / 2 \ln \left(h v_{0} /(k T)\right)\right. \\
& \quad-1 / 2 \psi(n / 2-1 / 2)\} \Gamma(n / 2-1 / 2) / \Gamma(n / 2)
\end{aligned}
$$

Finally then, with $n=3$ and 2 respectively,

$$
\begin{aligned}
\alpha_{r}(\text { Maxwell })= & \frac{\sqrt{2} c h v_{0} A}{\sqrt{ }\left(m_{e} c^{2} k T\right)} \frac{2}{\sqrt{\pi}}\left\{\gamma_{1}+1 / 2 \gamma\right. \\
& \left.+1 / 2 \ln \left(h v_{0} /(k T)\right)\right\} \\
\alpha_{r}(\text { flattened })= & \frac{\sqrt{2} c h v_{0} A}{\sqrt{ }\left(m_{e} c^{2} k T\right)} \sqrt{\pi}\left\{\gamma_{1}+1 / 2 \gamma\right. \\
& \left.+1 / 2 \ln \left(h v_{0} /(k T)\right)+\ln 2\right\} .
\end{aligned}
$$

For the Maxwell distribution, we can compare with the more accurate results of Spitzer. ${ }^{4} \mathrm{He}$ integrated (1) term by term over electron velocities and summed the resulting series numerically. He quotes values of a function $\phi(\beta)$ defined by

$$
\begin{aligned}
\alpha_{r} & =\beta \phi(\beta) 2 A V\left(2 k T /\left(m_{e} \pi\right)\right) \\
\beta & =h v_{0} /(k T)
\end{aligned}
$$

From (17), with $\gamma_{1}=\gamma$,

$$
\begin{aligned}
\phi(\beta) & =\ln \sqrt{\beta}+3 \gamma / 2 \\
& \doteqdot \ln (2.377 \sqrt{\beta})
\end{aligned}
$$

This accurately reproduces Spitzer's values for large $\beta$ (low electron temperature) and even for $\beta=5(k T=2.72 \mathrm{eV}$ when $Z=1)$, it is too low by less than $2 \%$. 


\section{THIRD APPROXIMATION}

However, so far we have made the approximation (2). To judge the accuracy of this we compare the results of (5) and (6) with values quoted by Bates et al. ${ }^{3}$ The comparison in Table I shows errors of $10-15 \%$. The work of Seaton ${ }^{3}$ suggests that considerable improvement results on replacing (6) by

$$
\sigma=A\left(\frac{h \nu_{0}}{E}\right)\left\{\ln \sqrt{\frac{h \nu_{0}}{E}}+\gamma_{1}+\gamma_{2}\left(\frac{E}{h \nu_{0}}\right)^{1 / 3}\right\},
$$

with

$$
\gamma_{1}=0.1402 \quad \gamma_{2}=0.525 .
$$

This is indeed so, as shown in the table.

The expression (23) is again readily integrated over thermal distributions; (16) is replaced by

$$
\begin{aligned}
& \left\{\ln \sqrt{\frac{h \nu_{0}}{k T}}-\frac{1}{2} \psi\left(\frac{n}{2}-\frac{1}{2}\right)+\gamma_{1}\right. \\
& \left.\quad+\gamma_{2} \frac{\Gamma\left(\frac{n}{2}-\frac{1}{6}\right)}{\Gamma\left(\frac{n}{2}-\frac{1}{2}\right)}\left(\frac{k T}{h v_{0}}\right)^{1 / 3}\right\} \frac{\Gamma\left(\frac{n}{2}-\frac{1}{2}\right)}{\Gamma\left(\frac{n}{2}\right)},
\end{aligned}
$$

so that

$$
\begin{aligned}
\alpha_{r}(\text { Maxwell })= & \frac{\sqrt{2} c h \nu_{0} A}{\sqrt{m_{e} c^{2} k T}} \frac{2}{\sqrt{\pi}}\left\{\gamma_{1}+\frac{1}{2} \gamma\right. \\
& +\Gamma\left(\frac{4}{3}\right) \gamma_{2}\left(\frac{k T}{h \nu_{0}}\right)^{1 / 3} \\
& \left.+\ln \sqrt{\frac{h \nu_{0}}{k T}}\right\}, \\
\alpha_{r}(\text { flattened })= & \frac{\sqrt{2} c h \nu_{0} A}{\sqrt{m_{e} c^{2} k T}} \sqrt{\pi}\left\{\gamma_{1}+\frac{1}{2} \gamma\right. \\
& +\Gamma\left(\frac{5}{6}\right) \frac{\gamma_{2}}{\sqrt{\pi}}\left(\frac{k T}{h \nu_{0}}\right)^{1 / 3} \\
& \left.+\ln \left(2 \sqrt{\frac{h \nu_{0}}{k T}}\right)\right\} .
\end{aligned}
$$

TABLE I

Comparison of Different Calculations of the Capture Cross Section

\begin{tabular}{lcccc}
\hline$h v_{0} / E$ & 49.0 & 100 & 196 & 400 \\
$E(Z=1, e V)$ & 0.278 & 0.136 & 0.069 & 0.034 \\
$\sigma\left(\right.$ Bates et al $\left.{ }^{3}\right)$ & 23.0 & 53.7 & 119 & 272 \\
$\quad\left(10^{-21} \mathrm{~cm}^{2}\right)$ & & & & \\
$\sigma[(5)(6)]$ & 26.1 & 60.8 & 133 & 302 \\
$\sigma\left[(23)\left(23^{\prime}\right)\right]$ & 23.1 & 53.9 & 119 & 271 \\
\hline
\end{tabular}

Numerically, with $k T$ in $e V, Z=1$,

$$
\begin{aligned}
\alpha_{r}(\text { Maxwell })= & \frac{1.92}{\sqrt{k T}}\left\{\ln \frac{5.66}{\sqrt{k T}}\right. \\
& \left.+0.196(k T)^{1 / 3}\right\} 10^{-13} \mathrm{~cm}^{3} / \mathrm{sec},
\end{aligned}
$$

$$
\begin{aligned}
\alpha_{r}(\text { flattened })= & \frac{3.02}{\sqrt{k T}}\left\{\ln \frac{11.32}{\sqrt{k T}}\right. \\
& \left.+0.140(k T)^{1 / 3}\right\} 10^{-13} \mathrm{~cm}^{3 /} / \mathrm{sec} .
\end{aligned}
$$

Seaton $^{3}$ gives a formula equivalent to (27). His error estimates imply accuracy to within a few per cent for $k T<1 \mathrm{eV}$. The formula is good within one per cent in comparison with results quoted by Massey et al. ${ }^{3}$ (their table 14.4) for $0.02 \mathrm{eV} \leq k T \leq 5 \mathrm{eV}$. We suppose that (28) will be of comparable accuracy.

Simple approximations to these formulae are

$\alpha_{r}($ Maxwell $)=3.79(k T)^{-0.678} 10^{-13} \mathrm{~cm}^{3} / \mathrm{sec}$

$\alpha_{r}($ flattened $)=7.88(k T)^{-0.645} 10^{-13} \mathrm{~cm}^{3} / \mathrm{sec}$.

These reproduce (27) and (28) to within one per cent for

$$
0.07<k T<0.70
$$

and to within ten per cent for

$$
0.01<k T<3.0 \text {, }
$$

with $k T$ always in $e V$.

These formulae, together with (8), apply in the first instance in the frame of reference in which the electron gas is at rest. Retaining formulae 
(27)-(30) with $T$ defined in the rest system of the electron gas, in the laboratory system the mean capture rate per proton is

$$
\alpha_{r} n_{e} d \gamma^{-2}
$$

where $n_{e}$ is the electron density in the lab system, $\left.\gamma=1 / \sqrt{\left(1-V_{0}^{2} / c^{2}\right.}\right), \gamma=\left(1-V_{0}{ }^{2} / c^{2}\right)^{-1 / 2}, V_{0}$ is the mean electron velocity in the lab, and $d$ is the fraction of ion orbit to which cooling is applied.

\section{ACKNOWLEDGMENTS}

We thank P. Møller-Petersen and H. Poth for useful remarks, and L. Bracci and G. Fiorentini for helpful correspondence.

$$
\text { APPENDIX } p+\bar{p} \rightarrow(p \bar{p})+\gamma
$$

We can also compare (23) with calculations ${ }^{7}$ on the radiative capture of antiprotons by protons, taking account of the Coulomb interaction only between the particles. To adapt the formulae, we have to replace $m_{e}$ by the reduced mass

$$
m_{e} \rightarrow \mu=m_{p} / 2
$$

in $A$ and $h \nu_{0}$, and interpret $E$ as center-of-masssystem kinetic energy. Numerically then, with $E$ in $e V$,

$$
\begin{aligned}
\sigma= & 3.1210^{-24} \frac{1}{E}\left\{\ln \sqrt{\frac{12500}{E}}\right. \\
& \left.+0.14+0.525\left(\frac{E}{12500}\right)^{1 / 3}\right\} \mathrm{cm}^{2}
\end{aligned}
$$

Brazzi, Fiorentini and Pitzurra ${ }^{7}$ give the cross section in units of the square of the Bohr radius

$$
a_{0}^{2}=0.28010^{-16} \mathrm{~cm}^{2} .
$$

They measure energy in units of

$$
\alpha^{2} m_{e} c^{2}=27.2 e V .
$$

With

$$
E=T \alpha^{2} m_{e} c^{2}
$$

(A1) becomes

$$
\begin{aligned}
10^{8} \sigma / a_{0}{ }^{2}= & \frac{0.409}{T}\left\{\ln \sqrt{\frac{459}{T}}\right. \\
& \left.+0.14+0.525\left(\frac{T}{459}\right)^{1 / 3}\right\} .
\end{aligned}
$$

This agrees with the numerical results of Brazzi et al. to within the accuracy (some ten per cent) of their calculations (private communication), which are for the range

$$
1>T>10^{-3} \text {. }
$$

We would expect (A2) to be quite accurate in this range, where $E$ is indeed small compared with the relevant

$$
\begin{aligned}
h \nu_{0} & =\alpha^{2} m_{p} c^{2} / 4 \\
& =459 \alpha^{2} m_{e} c^{2} .
\end{aligned}
$$

\section{REFERENCES}

1. G. I. Budker, At. Energ. 22, 346 (1967).

2. G. I. Budker et al., Particle Accelerators 7, 197 (1976).

3. D. R. Bates et al., Proc. R. Soc. London A170, 322 (1939);

A. Burgess, Mon. Not. R. Astron. Soc. 118, 477 (1958); M. J. Seaton, Mon. Not. R. Astron. Soc. 119, 81 (1959); H. S. W. Massey et al., Electronic and Ionic Impact Phenomena (Oxford, 1969), Vol. 2, Ch. 14.

4. L. Spitzer, Physics of Fully Ionized Gases (Interscience, N.Y., 1956)

5. Handbook of Mathematical Functions, M. Abramowitz and I. Stegun, Eds. (Dover, N.Y., 1973).

6. G. I. Budker and A. N. Skrinsky, Usp. Fiz. Nauk. 124, 561 (1978) [Sov. Phys. Usp. 21, 277 (1978)].

7. L. Bracci et al., Phys. Lett. 85B, 280 (1979). 